\title{
Depression and cardiovascular disease: the end of simple models ${ }^{\dagger}$
}

Peter de Jonge and Annelieke M. Roest

\section{Summary}

In this editorial, we propose that the association between depression and cardiovascular disease may be

conceptualised as a continuous, bidirectional process that originates in youth. The paper by Åberg and colleagues

in this issue adds to this literature showing that low cardiovascular fitness at adolescence increases the risk of future depression.

\section{Declaration of interest}

None.
Peter de Jonge (pictured) is a psychologist and Professor of Psychiatry. He leads a research line on affective disorders and somatic diseases and publishes particularly on depression and cardiovascular disease. Annelieke Roest is a psychologist and works as a postdoctoral researcher in the Department of Psychiatry at the University of Groningen.

Depression and ischaemic heart disease are not independent entities. It has been demonstrated that depression is associated with incident cardiovascular morbidity and mortality, ${ }^{1}$ and that depression after myocardial infarction is prospectively associated with an increased risk of adverse cardiac outcomes. ${ }^{2}$ Although this latter finding has led researchers to believe that this reflects a relatively simple causal effect of depression on cardiovascular disease and its progression, by now the status of that hypothesis is uncertain: several large-scale attempts in which depression in cardiac patients was treated have concluded that no positive effects on cardiac outcomes could be attributed to psychiatric treatment. ${ }^{3}$

The elegant study by Åberg and colleagues in this issue of the British Journal of Psychiatry ${ }^{4}$ represents the other side of the coin, emphasising that the association between depression and cardiovascular disease may develop in the opposite direction. In a very large sample of young men enlisted for military service, the authors showed that cardiovascular fitness predicted the onset of serious depression in adulthood. By controlling for several confounders they made it less plausible that the observed effects were the result of shared risk factors such as obesity, low level of parental education, and familial influences. The suggestion of cardiovascular parameters affecting depression is not new in itself and falls well within a whole line of research such as the vascular depression hypothesis in which vascular factors are held responsible for the development of depression in older adults. Empirically, it is known that depression prevalence is considerably higher in patients with cardiovascular disease than in the general population. However, the study by Åberg et al goes well beyond these findings, as the authors demonstrate that already in young healthy men who are free of any form of clinical or subclinical cardiovascular disease, lower cardiovascular fitness as assessed during ergometer cycling is associated with future risk of incident depression. Thus, the authors make a compelling case for the depressogenic effects of low cardiovascular fitness even in the absence of confounding factors related to cardiovascular disease.

In this editorial we make the case that simple chicken-or-egg models in which depression causes cardiovascular disease or vice

'See pp. 352-359, this issue. versa in our opinion will turn out to be inadequate to address all scientific data in this field. The available literature as a whole may best be summarised with a hypothetical model describing the association between depression and cardiovascular disease as the outcome of two intertwined, mutually reinforcing disorders of which the roots may lie already in youth. The study by Ảberg and colleagues adds substantially to the body of literature supporting this notion using an exceptionally strong research design.

\section{How does this translate to clinical practice?}

Based on the study by Åberg et al, researchers with clinical ambitions may start to develop interventions aimed at cardiovascular parameters in an attempt to prevent or ameliorate depression, now that the reverse strategy has turned out largely unsuccessful. However, we believe such an approach is likely to fail as well. Instead, we favour an approach in which the complex nature of the association between depression and cardiovascular disease is taken well into account, based on the following reasoning.

First, information on mechanisms underlying the relationship between cardiovascular disease and depression, and therefore information on specific targets for intervention, is incomplete. This is problematic as ideally this type of research would provide the targets for effective interventions. In the literature, numerous potentially mediating mechanisms have been proposed and these include psychophysiological factors such as reduced heart rate variability, inflammatory processes, disturbed platelet function and hypothalamus-pituitary-adrenal (HPA) axis dysfunction, and behavioural factors such as physical inactivity, unhealthy diet, smoking and poor medication adherence. ${ }^{5}$ As depression and cardiovascular disease are both multifactorial and heterogeneous disorders, it is unlikely that any specific factor will explain a substantial part of the association between depression and cardiovascular disease. The literature in this field is still limited, as the abovementioned potential mediators are mostly studied in isolation while in reality they are all interrelated. Furthermore, the directions of potential mediational pathways are far from clear. In principle, these mechanisms are largely non-specific to the direction of the association, and may explain both cardiotoxic effects of depression and depressogenic effects of cardiovascular fitness. For example, depressive symptoms may lead to inflammation, but might also be the result of inflammatory processes. The same holds for behavioural factors such as physical activity and obesity. Directions of effects may even differ between individuals. Finally, genetic factors may underlie the development of both cardiovascular disease and depression, 
representing yet another kind of mediation, namely by a factor that was already present before depression and cardiovascular disease were. In sum, we still know little of the nature of the association between the two intertwined disorders, and there is little to support that a one-size-fits-all approach will be the solution to improve the prognosis of the two.

A second factor that adds to the complexity of translating these findings into effective interventions is the heterogeneity of the depression construct. Since its operationalisation in the DSM-III, numerous attempts have been made to detect relevant depression subtypes in terms of severity, symptom patterns and aetiologies. In cardiac patients, a differentiation in somatic and cognitive symptom dimensions of depression is often reported in which the somatic dimension is more strongly predictive of cardiac prognosis. ${ }^{6}$ Notably, symptom dimensions of distress also appear to be differentially related to potential mechanisms (e.g. heart rate variability and HPA axis dysregulation), with somatic symptoms being more intertwined with cardiovascular disease than cognitive symptoms of depression. ${ }^{7}$ This line of research suggests that underlying mechanisms linking depression and cardiovascular disease may be differentially involved across individuals. To positively influence depression and/or cardiovascular disease, different treatments may thus be required depending on depression subtype and mediating factors. Although this field of research is rather young and inconclusive, we feel that it holds promise for addressing the depression-cardiovascular disease association in clinical care.

\section{The solution: no more simple models}

We believe that simple models with respect to aetiology and thus treatment of depression or cardiovascular fitness will turn out to be inadequate and even hamper progress in this field. New models are needed in which the bidirectionality of the association, heterogeneity of depression, interdependency of mediators and inter-individual heterogeneity in the association is accounted for. Depression and cardiovascular disease are highly intertwined diseases which continuously influence each other. The interindividual heterogeneity of the association implies that, for instance, an underlying process such as atherosclerosis-based inflammation might lead to cardiovascular disease followed by a higher risk of depression in one individual, and depression followed by a higher risk of heart disease in another. In a third individual, atherosclerosis may even result from depression and subsequent poor lifestyle. To deal with this in clinical care, and as opposed to previously proposed simple models, we argue for an alternative research approach based on the following foundations.

(a) Research to date supports bidirectional associations between depression and cardiovascular parameters throughout the life span. Scientific progress on concepts that are so much intertwined will not be achieved by clinging to simple hypotheses stressing only one side of the story. Instead of treating one disorder in order to improve prognosis of the other, we need to treat both simultaneously.

(b) The massive interdependencies between physiological and behavioural mediators explaining the association between depression and cardiovascular disease suggest that networks of effects may give a far more complete representation of reality. In addition, research should take into account potential bidirectional effects of mechanisms. This argues for interventions that are relatively unselective, i.e. affecting several mechanisms simultaneously.

(c) The heterogeneity of the depression construct suggests that any attempt to improve cardiovascular parameters in order to influence depression, or to reduce depression in order to improve cardiovascular parameters, should be adjustable to the level of individuals.

Collaborative care and personalised medicine, in which depression and cardiovascular disease are not conceptualised as separate entities and in which interventions can be adjusted to the stages of the disease, in principle might offer the opportunity to address these issues. Lifestyle interventions including exercise therapy will likely play an important role in such programmes as they have the potential to improve depression and cardiovascular fitness simultaneously and may affect several of the proposed mediators simultaneously. Research to date has shown that collaborative care may be an effective tool in addressing depression in the general population, ${ }^{8}$ but also depression as a comorbid disorder in patients with somatic diseases. ${ }^{9}$ Still, collaborative care is not a golden solution in itself, and its active ingredients are far from being fully understood. ${ }^{10}$ The true effectiveness of collaborative care in specific patients will depend on how well clinicians are able to adjust the interventions to the needs of individuals, based on a thorough understanding of the association between depression and cardiovascular fitness within individuals. Needless to say perhaps, this may hold for the depression-cardiovascular disease link as well as for the link between depression and other medical conditions.

Peter de Jonge, PhD, Department of Psychiatry, University of Groningen; Annelieke M. Roest, PhD, University Medical Center Groningen, The Netherlands

Correspondence: Peter de Jonge, Department of Psychiatry, University of Groningen, Hanzeplein 1, P.O.Box 30001, Groningen 9700 RB, The Netherlands. Email: peter.de.jonge@med.umcg.nl

First received 19 Mar 2012, final revision 23 May 2012, accepted 23 Jul 2012

\section{References}

1 Van der Kooy K, van Hout H, Marwijk H, Marten H, Stehouwer C, Beekman A. Depression and the risk for cardiovascular diseases: systematic review and meta-analysis. Int J Geriatr Psychiatry 2007; 22: 613-26.

2 Meijer A, Conradi HJ, Bos EH, Thombs BD, van Melle JP, de Jonge $P$. Prognostic association of depression following myocardial infarction and cardiovascular events: a meta-analysis of 25 years of research. Gen Hosp Psychiatry 2011; 33: 203-16.

3 Thombs BD, de Jonge P, Coyne JC, Whooley MA, Frasure-Smith N, Mitchell AJ, et al. Depression screening and patient outcomes in cardiovascular care: a systematic review. JAMA 2008; 300: 2161-71.

4 Åberg MAI, Waern M, Nyberg J, Pedersen NL, Berg Y, Åberg ND, et al. Cardiovascular fitness in males at age 18 and risk of serious depression in adulthood: Swedish prospective population-based study. Br J Psychiatry 2012; 201: 352-9.

5 De Jonge P, Rosmalen JG, Kema IP, Doorbos B, van Melle JP, Pouwer F, et al. Psychophysiological biomarkers explaining the association between depression and prognosis in coronary artery patients: a critical review of the literature. Neurosci Biobehav Rev 2010; 35: 84-90.

6 De Jonge P, Ormel J, van den Brink RHS, van Melle JP, Spijkerman TA, Kuijper $A$, et al. Symptom dimensions of depression following myocardial infarction and their relationship with somatic health status and cardiovascular prognosis. Am J Psychiatry 2006; 163: 138-44.

7 Ormel J, de Jonge P. Unipolar depression and the progression of coronary artery disease: toward an integrative model. Psychother Psychosom 2011; 80: $264-74$

8 Gilbody S, Bower P, Fletcher J, Richards D, Sutton AJ. Collaborative care for depression: a cumulative meta-analysis and review of longer-term outcomes. Arch Intern Med 2006; 166: 2314-21.

9 Bower P, Gilbody S, Richards D, Fletcher J, Sutton A. Collaborative care for depression in primary care. Making sense of a complex intervention: systematic review and meta-regression. Br J Psychiatry 2006; 189: 484-93.

10 Katon WJ, Lin EH, Von Korff M, Ciechanowski P, Ludman EJ, Young B, et al. Collaborative care for patients with depression and chronic illnesses. N Engl J Med 2010; 363: 2611-20. 